\title{
Multiple Description Wavelet-Based Image Coding Using Iterated Function System
}

\author{
Jie Yang \\ School of Science, Beijing Information Science and Technology University, Beijing 100192, China \\ Correspondence should be addressed to Jie Yang; zezhongy@163.com
}

Received 17 December 2012; Revised 17 February 2013; Accepted 18 February 2013

Academic Editor: Wang Xing-yuan

Copyright (c) 2013 Jie Yang. This is an open access article distributed under the Creative Commons Attribution License, which permits unrestricted use, distribution, and reproduction in any medium, provided the original work is properly cited.

\begin{abstract}
Recent literature highlights the multiple description coding (MDC) as a promising method to solve the problem of resilient image coding over error-prone networks, where packet losses occur. In this paper, we introduce a novel multiple description wavelet-based image coding scheme using fractal. This scheme exploits the fractal's ability, which is to describe the different resolution scale similarity (redundancy) among wavelet coefficient blocks. When one description is lost, the lost information can be reconstructed by the proposed iterated function system (IFS) recovering scheme with the similarity and some introduced information. Compared with the referenced methods, the experimental results suggest that the proposed scheme can achieve better performance. Furthermore, it is substantiated to be more robust for images transmission and better subjective quality in reconstructed images even with high packet loss ratios.
\end{abstract}

\section{Introduction}

Transmission of compressed images over unreliable networks has proven to be a significant challenge. The main problem is the rapid degradation in the reconstructed image quality due to packet loss, which is unavoidable on the networks, such as the Internet. Hence, it is important to propose an effective method to protect compressed images information transmitted over unreliable networks.

Recently, MD coding has emerged as an attractive framework for robust transmission over unreliable networks. It can efficiently combat packet loss without any retransmission thus, satisfying the demand of real-time services and relieving the network congestion [1]. In MD coding, two or more bitstreams called descriptions of the same image are generated, which can be independently decoded. At the same time, the descriptions should carry correlated information (redundancy). The correlated information is beneficial in the case of single description reception in that it helps the estimation of the missing description from the received one. So, a minimum fidelity in the reconstruction can be guaranteed at the receiver when only one channel works.
When more channels work, a higher fidelity reconstruction can be yielded by combining the descriptions. However, an extra rate is necessary to encode correlated information, which impairs the rate-distortion performance. In many practical situations, only two descriptions of the same image are generated; throughout this paper, we will make this assumption.

Lots of MD coding techniques have been developed using different strategies for coding variant data like speech, audio, image, and video. One of the most classical methods is multiple description scalar quantization (MDSQ) [2], which was successfully applied in image coding [3]. The pairwise correlating transform (PCT) was exploited in [4]. Multiple description lattice vector quantization (MDLVQ) has also shown promising results for image coding [5]. Among many others, a feature-oriented multiple description wavelet-based image coding was recently presented in [6]. In that method, a wavelet image is partitioned into two parts, for example, part 1 and part 2, each of which goes through a fine coding as well as a coarse coding. Then, one description is formed by concatenating the finely coded part 1 and the coarsely coded part 2, while the other description is complementarily 
generated by joining the coarsely coded part 1 and the finely coded part 2. Similar MD coding schemes can also be found in $[7,8]$.

From the above analysis, the most MD coding approaches are based on traditional coding methods, such as quantization, JPEG, and wavelet. These methods always exploit the redundancy in the neighboring pixels or coefficients. This redundancy is partly added to the descriptions for the recovering in case of some descriptions missed. But the redundancy among coefficient blocks at different resolution scales exists in wavelet domain, and this kind of redundancy is not used in MDC. Fortunately, fractal coding (FC) is an intensively studied image coding method during recent decades [9-11], which can exploit this kind of redundancy.

In this paper, we attempt to design an MD waveletbased image coding method using fractal. Fractal coding has some advantages, such as high decompression speed, low compression bit rate, and decompression resolution independence. The basic principle of FC is to find an IFS whose fixed point approximates to the original data. Therefore, for the original data, the compressed data is an IFS after FC. As discussed previously in [12-14], FC relies on selfsimilarity of images. The wavelet transform is a tool for demonstrating the scale invariance of edges. It is obvious that the subbands in successive wavelet levels are similar. Therefore, it is natural to use FC in the wavelet domain. The relationship between fractal and transform-based coders has been recently investigated in [15-19]; Rinaldo and Calvagno [16] proposed a predictive pyramid coder (PPC) by exploring the interscale redundancy. Davis [17] showed that the fractal contractive mapping could be considered as a prediction operation in the wavelet domain. And Xing-Yuan et al. [2025] proposed a series of methods to improve the coding speed of FC, which is another research direction in FC.

In our study, the wavelet transform decomposes the original image into different resolution subbands. At each decomposition level, four subbands are produced. They are named approximation subband (low-pass version) and detail subbands (high-pass version). Using the primary descriptions generation method, two subsets are generated at the encoder. For each subset, we use the extended range blocks fractal method in [26] to compress the approximation subband and prediction fractal method [17] to compress the detail subbands. So, IFS in wavelet domain is constructed as a description, and the difference between the collage and original wavelet coefficients is also quantized and entropy coded in order to enhance the coding performance. Meanwhile, redundancy is introduced into each description. At the decoder, an IFS recovering scheme using the similarity in intra- and intersubbands is presented to reconstruct IFS when only one description is received. The novelty of this paper is (1) compared with the classical MDSQ method; iterated function system is used to replace the SQ in MD coding so that a better performance of MD coder can be achieved. (2) Further, s multiple channel generation and reconstruction for MD coding based on IFS are designed.

The rest of this paper is organized as follows. In Section 2, basic idea and related techniques are formulated. In Section 3, the design method of multiple description using fractal is proposed. The experimental results are given in Section 4. We conclude the paper in Section 5.

\section{Basic Idea and Related Techniques}

2.1. Basic Idea. $\mathrm{MD}$ coding can tolerate loss from the estimation of missing data with help of a certain amount of introduced redundancy. So, the interests of MD coding are mainly two points; first, in what way the descriptions and redundancy should be created to benefit the lost data estimation; second, how can we efficiently recover the lost data?

Therefore, in this paper, an MD wavelet-based image coding scheme using fractal is proposed. Wavelet is a popular tool for image analysis and compression. The different subbands of wavelet coefficients have similarity, which can be exploited by fractal coding methods. In summary, the proposed scheme partitions the mappings of IFS in wavelet domain with the checkerboard pattern and recovers the lost mappings using IFS recovering schemes.

2.2. FC and IFS. FC partitions data image $x_{\text {orig }}$ of size $N \times N$ into nonoverlapping range blocks $R_{j}$ of a predefined size $B \times B$, where $B \in 2^{Z^{+}}$. Then a domain pool (DP) is created to take all the square blocks of size $2 B \times 2 B$. For each range block $R_{j}$, a mapping $\omega_{j}(D)=s_{j} \times D+o_{j}$ is constructed to satisfy the following minimization equation:

$$
\begin{aligned}
& \left(D_{i}, \omega_{j}\right)=\arg \min _{\left(D_{i}, \omega_{i}\right)}\left\|\omega_{j}\left(D_{i}\right)-R_{j}\right\|_{2} \\
& \text { s.t. } D_{i} \in \text { DP for } j=1,2, \ldots, \frac{N^{2}}{B^{2}},
\end{aligned}
$$

where $D_{i}$ is the $i$ th domain block in DP and arg means finding the best parameters $\left(D_{i}\right.$ and $\omega_{j}$ here $)$ to fit the minimum problems. \|\|$_{2}$ is the norm of vector.

Note that the range-domain matching process initially consists of a shrinking operation in each domain block that averages its pixel intensities forming a block of size $B \times B$. Then, for each range block $R_{j}$, we can find the mapping $\omega_{j}$ and a domain block $D_{i}$ to satisfy (1). The $s_{j}$ and $o_{j}$ in $\omega_{j}$ are called scale and offset parameters, respectively, corresponding to the $j$ th range block. And solving the minimization problem (1), the optimal affine parameters can be obtained

$$
s_{j}=\frac{\left\langle R_{j}-\overline{r_{j}} I, D_{i}-\overline{d_{i}} I\right\rangle}{\left\|D_{i}-\overline{d_{i}} I\right\|_{2}^{2}}, \quad o_{j}=\overline{r_{j}}-s_{j} \times \overline{d_{i}},
$$

where $I$ is a matrix whose elements are all ones, $\bar{r}_{j}, \bar{d}_{i}$ are the average pixel intensity of the range block and the contracted domain block, respectively. And $\langle\cdot, \cdot\rangle$ is inner product, and in this paper, $s_{j}$ and $o_{j}$ are real numbers. So in FC, the parameters that basically need to be placed in the encoded bit stream are $s_{j}, o_{j}$, position of domain block. And we use the sliding windows method to find the best domain block $D_{i}$ for each range block. 
Assuming that $\omega_{j}$ is contractive in the pixel intensity shifting, that is,

$$
\left|s_{j}\right|<1, \quad \forall j,
$$

then an IFS $\Gamma$ can be constructed by combining all $\omega_{j}$ :

$$
\Gamma=\omega_{1} \circ \omega_{2} \circ \cdots \circ \omega_{N^{2} / B^{2}},
$$

where $\circ$ is series operation for different $\omega$ s.

The fixed point theory and the collage theory are two basic theorems for IFS.

2.2.1. The Fixed Point Theory. Let $(X, d)$ denote a metric space, where $d$ is a given distortion metric. If a transformation $\Gamma$ satisfies (5), we call $\Gamma$ a contractive IFS in $(X, d)$.

For any two points $\mu, v \in X$,

$$
d(\Gamma(\mu), \Gamma(\nu)) \leq s \cdot d(\mu, \nu),
$$

where $-1<s=\max \left(s_{j}\right)<1, s$ is called contraction factor.

For any contractive transformations, there must be a fixed point $x^{*}$, that is,

$$
\Gamma\left(x^{*}\right)=x^{*},
$$

and the fixed point can be obtained from any point $x$, such that

$$
\lim _{n \rightarrow \infty} \Gamma^{n}(x)=x^{*}
$$

2.2.2. The Collage Theory. Let $(X, d)$ be a metric space, a contractive IFS $\Gamma: X \rightarrow X$ with contractive factor $s$ and fixed point $x^{*}$ is in this space. Then, for any $x \in X$,

$$
d\left(x, x^{*}\right) \leq \frac{1}{1-s} d(x, \Gamma(x)) .
$$

The fixed point theory ensures that IFS $\Gamma$ has a unique fixed point, and the fixed point can be found by iteration of $\Gamma$. According to the collage theory, $x_{\text {orig }}$ can be approximately reconstructed by applying $\Gamma$ on any initial data image $x$ iteratively if $\Gamma\left(x_{\text {orig }}\right) \approx x_{\text {orig }}$. If $\Gamma$ can be stored compactly, then it is called the compressed data of $x_{\text {orig. }}$. The process of constructing the mappings of IFS can be seen in Figure 1.

The decoder is simple; it is shown in [12] that if IFS is performed iteratively, beginning from an arbitrary data image of equal size, the result will be an attractor resembling the original data image at the chosen resolution.

\subsection{Wavelet-Based Image Coding Using Fractal with Redun-} dancy. In our study, in case of recovering the lost mappings, a wavelet coefficient image is compressed by the fractal coder with redundancy. It is well known that the different wavelet coefficient subbands have a different kind of similarity, for example, the approximation subband has much similarity in intrasubband and the detail subbands have much similarity in intersubbands. Therefore, in the proposed method, the different FCs are selected according to the different subbands. In the approximation subband, we use the extended range block FC method. And prediction fractal coding method is used in detail subbands.

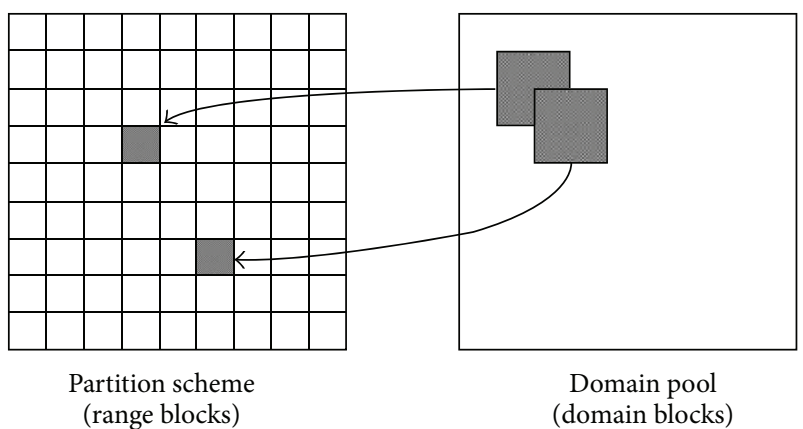

FIGURE 1: Constructing the mappings of IFS.

2.3.1. The Extended Range Block Fractal Coding. The basic idea of the extended range block fractal coding is sending the image twice with the same amount of data as the single image and using double-sized range blocks and a new criterion to determine the domain block [26].

This method extends the original range blocks into new range blocks which are overlapped just like Figure 2. The new range block consists of two parts. The white part is called basic range block, and the gray part is called extended range block. In Figure 2, the approximation subband is firstly partitioned into nonoverlapping basic range blocks. And each basic range block extends some pixels to construct extended range blocks. Each basic range block is overlapped by the extended range blocks from its neighboring basic range blocks. Let us take the basic range block $R$ as an example, $R$ is overlapped by the expanded block 1 from basic range block $R_{u}$, the expanded block 2 from basic range block $R_{l}$, the expanded block 3 from basic range block $R_{d}$, and the expanded block 4 from basic range block $R_{r}$. So, all the basic range blocks can construct the approximation subband, and all the extended range blocks can construct the approximation subband too.

The process of finding an IFS with new range blocks is just like Section 2.2. Note that the criterion has a little bit changed. Different weights are used in different blocks to guarantee the decoded quality. So, the new criterion can be expressed as

$$
\begin{aligned}
& \left(D_{i}, \omega_{j}\right)=\arg \min _{\left(D_{i}, \omega_{j}\right)}\left\|g_{j} *\left(\omega_{j}\left(D_{i}\right)-R_{j}\right)\right\|_{2} \\
& \text { s.t. } D_{i} \in \mathrm{DP} \quad \text { for } j=1,2, \ldots, \frac{N^{2}}{B^{2}},
\end{aligned}
$$

where $R_{j}$ has two parts, one is the basic range block, the other is the extended blocks. $g_{j}$ is the weight for the extended range blocks. The weight for the basic range blocks is 1 .

2.3.2. Prediction Fractal Coding. We use prediction fractal coding [17] to compress the detail subbands, because the detail subbands have much similarity in intersubbands. Davis showed that the fractal contractive mapping could be considered as a prediction operation in the wavelet domain. That is, coefficients of higher subbands are predicted from those of the lower subbands. The isometry, scale, and prunes operator used in spatial fractal operators are still used in the wavelet domain. And the coding process is shown in Figure 3. 


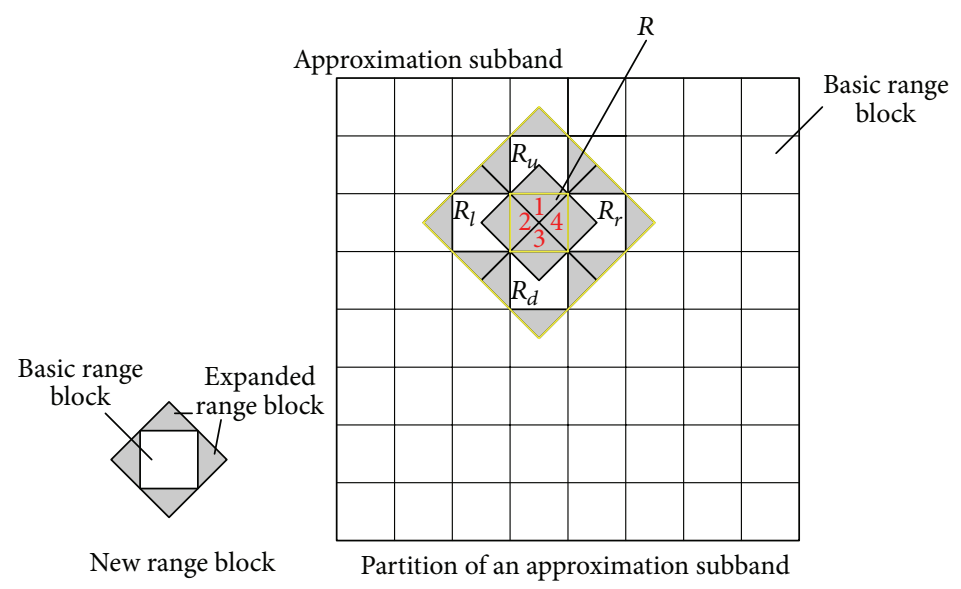

FIGURE 2: New range block and partition of extended range block FC.

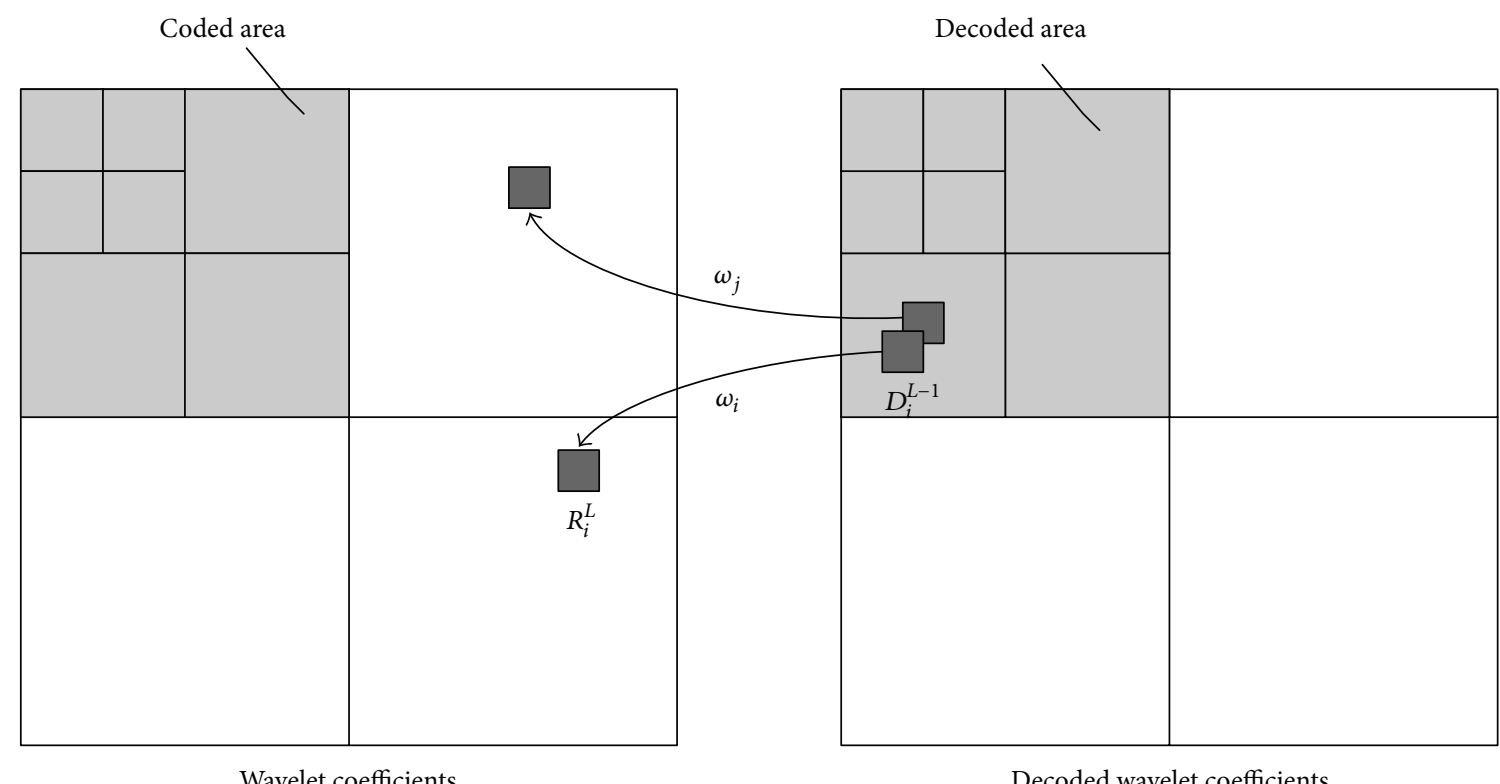

FIGURE 3: Coding process of prediction fractal coding method.

In Figure 3, the shadow area has already been coded (left) or decoded (right). For each range block in subband $L$, we find the best matched domain block in subband $L-1$ of decoded wavelet coefficients. So, the following equation must be satisfied:

$$
\begin{array}{r}
\left(D_{i}^{L-1}, \omega_{i}\right)=\arg \min _{\left(D_{i}^{L-1}, \omega_{i}\right)}\left\|\omega_{i}\left(D_{i}^{L-1}\right)-R_{i}^{L}\right\|_{2} \\
\text { for } i=1,2, \ldots, \frac{N^{2}}{B^{2}},
\end{array}
$$

where $R_{i}^{L}$ is range block in coded subband $L, D_{i}^{L-1}$ is domain block in decoded subband $L-1$, and $\omega_{i}\left(D_{i}^{L-1}\right)=s_{i} * D_{i}^{L-1}$. And solving the minimization problem (10), the optimal affine parameters can be obtained

$$
s_{i}=\frac{\left\langle R_{i}^{L}, D_{i}^{L-1}\right\rangle}{\left\|D_{i}^{L-1}\right\|_{2}} .
$$

The difference between the original coefficients and decoded coefficients is quantized and transformed for improving the quality of decoded image.

2.4. Generating the Primary Descriptions. After wavelet transform, each subband is divided into a group of blocks, the block size may vary for different subbands, then these blocks are partitioned into two nonoverlapping subsets; the mappings corresponding to the blocks in subset are defined 


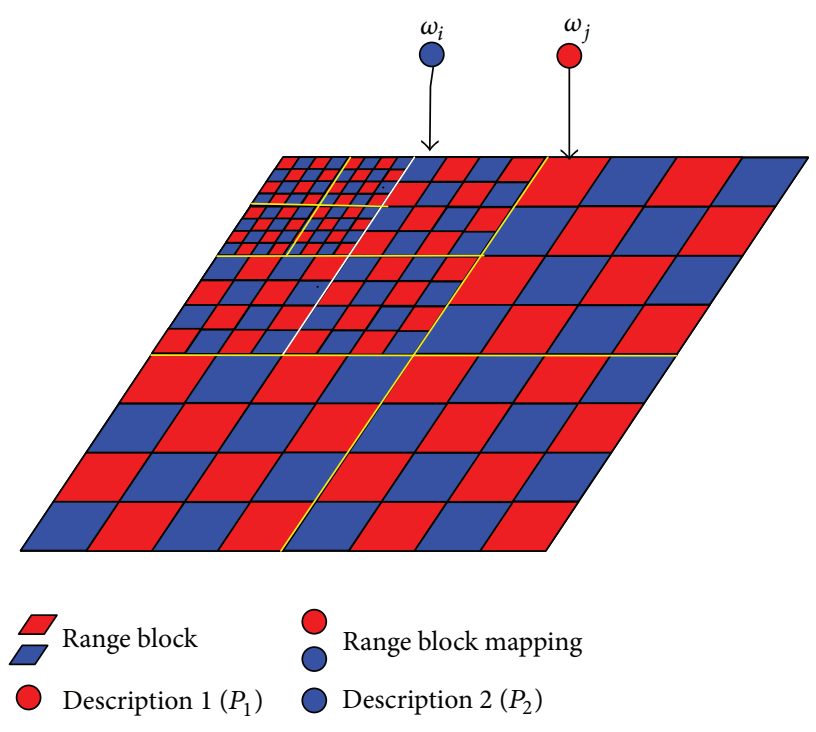

FIgURE 4: Primary description generation.

as primary descriptions $P_{i}(i=1,2)$. Figure 4 illustrates this decomposition. The range blocks are divided into two groups with checkerboard pattern. Thus, the mappings corresponding to the range blocks are divided into two groups. Each mapping group is assigned to one of the primary descriptions. In Figure 4 , the mapping $\omega_{j}$ is included in $P_{1}$ and $\omega_{i}$ is included in $P_{2}$.

Redundancy which is defined as correlated information $C_{i}(i=1,2)$ is appended to the primary descriptions. The correlated information is used for IFS reconstruction when only one description is received. $C_{i}(i=1,2)$ will guarantee the reconstructing quality when one description is lost, but it will also reduce achievable compression gains. In the next Section, we will explain how to generate a desired amount of correlated information for each description. By symmetry, the rest discussion is devoted for one description, while the other description can be done in the same manner.

\subsection{IFS Recovering Scheme}

2.5.1. IFS Recovery in Approximation Subband. In approximation subband, the extended range block fractal coding method is exploited to compress wavelet coefficients. When only one description is available, the received data is considered as an error IFS of the original image. Because the extended range blocks can cover the lost basic range blocks, we use the basic range blocks in received description and its extended range blocks as new range blocks. In this way, iteratively applying half mappings of IFS with the new range blocks to an initial data image, we can acheive the decoded subband. Note that, in each iterative step, the correlated information is added to the lost basic range blocks.

In Figure 5, the black block is the lost block and the gray block is extended range block. When one description is lost, the lost basic range blocks can be recovered by the extended range blocks in the other description.
When both descriptions are available, we use the basic range blocks as the range blocks. With the completed IFS, the decoded subband can be calculated just like the traditional fractal decoding process.

2.5.2. IFS Recovery in Detail Subbands. In detail subbands, prediction fractal image coding method is used to compress the wavelet coefficients. When only one description is available, half mappings of IFS are lost in the checkerboard pattern manner. So, the fractal extrapolation method is presented to estimate the lost mappings. This method exploits the similarity in intrasubbands and the introduced correlated information (redundancy).

In detail subbands, there is the similarity in intersubbands at the same direction. So in our study, the lost mapping in subband $L$ is estimated by the mapping parameters in subband $L-1$. Note that the range block and the domain block corresponding to the lost mapping in subband $L$ must have the same location with the estimation range block and the domain block in subband $L-1$ in spatial domain. In other words, the range blocks corresponding to the lost mapping and estimation mapping are included in the same wavelet tree, and the domain blocks of the lost mapping and the estimation are in the same wavelet tree too. Figure 6 is given to explain this fractal extrapolation method, and it is expressed by

$$
\bar{\omega}\left(D_{i^{\prime}}\right)=\omega\left(D_{i^{\prime}}\right)
$$

where $\bar{\omega}$ is the estimated mapping, $\omega$ is the range block mapping of lower subband in the same wavelet tree computed in Section 2.3.2. $D_{i^{\prime}}$ denotes the corresponding domain block of $D_{i}$.

For each estimated block, the correlated information is added on it.

\section{The Proposed MDC Scheme}

3.1. System Outline. The proposed MDC can be depicted in Figure 7 in which (a) is MD encoder and (b) is MD decoder. In Figure 7, Q1 means quantizer for channel 1. DQ1 means dequantizer for channel 1. Q2 means quantizer for channel 2. DQ2 means dequantizer for channel 2. DWT means digital wavelet transform and IDWT means inverse digital wavelet transform.

At the encoder, a given input image is decomposed by wavelet transform, and the wavelet coefficients are partitioned into two descriptions by the primary description generation method. In each description, for the approximation subband, extended range block fractal coding method is used to compress the approximation subband coefficients. we use prediction fractal image coding scheme to compress the detail subband. The detail has been given in Section 2 . With the correlated information $C_{i}$, the final descriptions are generated. At the decoder, if only one description is received, the side decoder is used to decode the image. When both descriptions are received, the central decoder is used to reconstruct the image. 


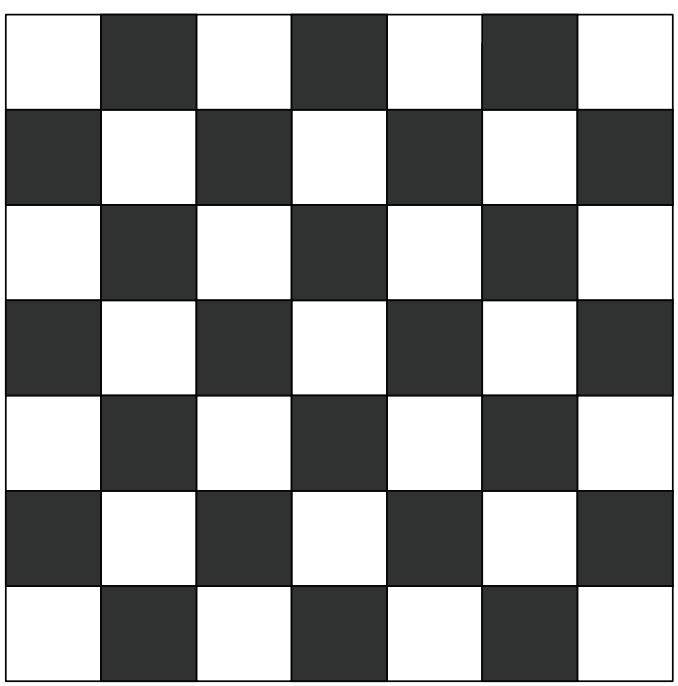

Approximation subband

Lost basic block

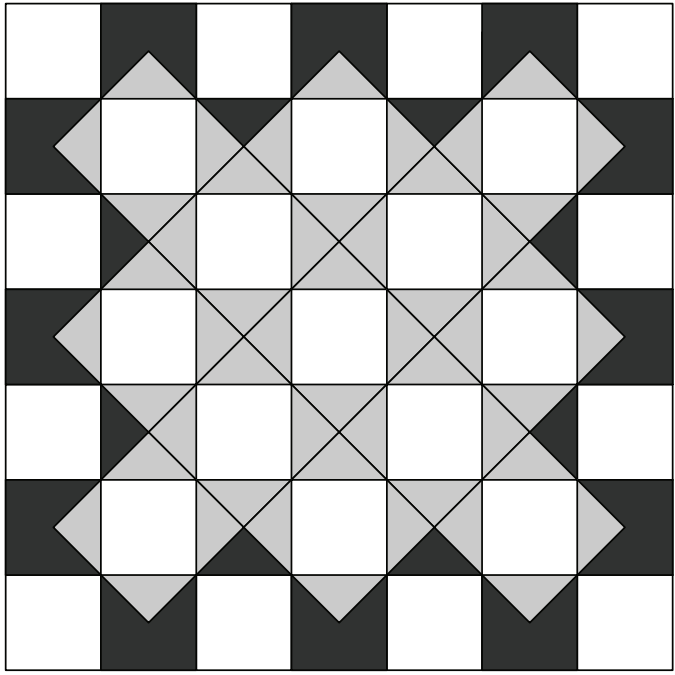

Extended approximation subband

Basic range block and

extended range block

FIGURE 5: Lost blocks are recovered by extended range blocks.

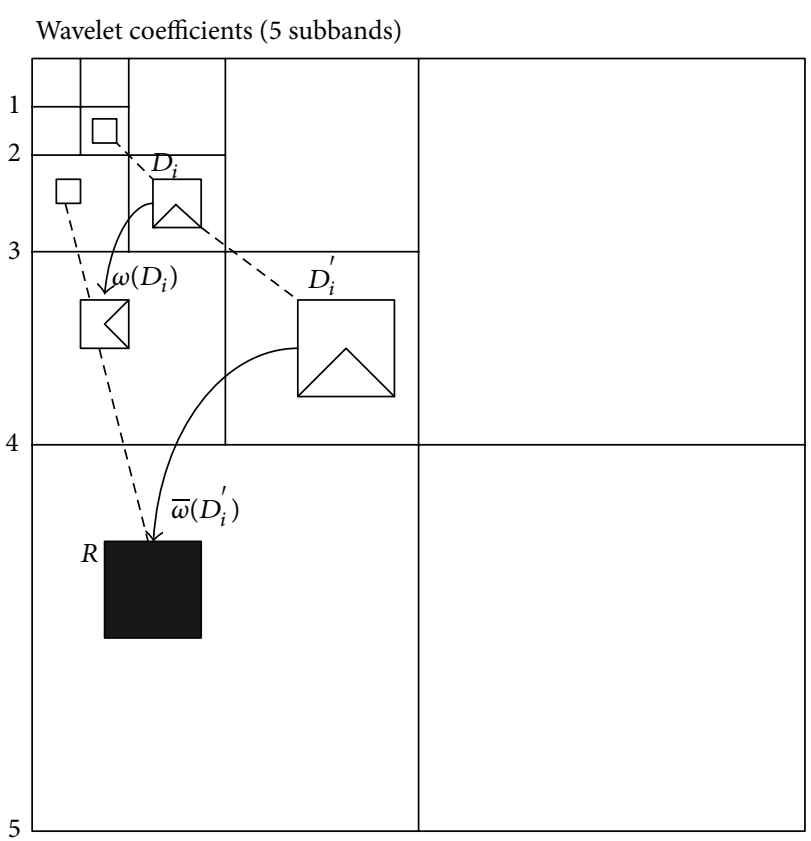

Lost block

$\checkmark$ Estimation block

Figure 6: Example of fractal extrapolation.

Let $R_{t}=2\left(R_{t}^{p}+R_{t}^{r}\right)$ be the target rate for both channels, where $R_{t}^{p}$ and $R_{t}^{r}$ denote the target rates for the primary description $P_{i}$ and the correlated information $C_{i}$. Assume $R^{p}$ denote the actual rate for the primary description $P_{i}$ and $R^{r}$ denote the actual rate for the correlated information $C_{i}$.
Let $D_{s}$ be the distortion between the decoded image and the original image in one description and let $D_{c}$ be the distortion of central decoded image and the original image.

In the proposed scheme, the central decoded image quality is directly dependent on $R^{p}$ and $R^{r}$. Hence, the problem for the central channel can be formulated as

$$
\begin{aligned}
& \min D_{c}\left(R^{p}, R^{r}\right) \\
& \text { subject to } R^{p}+R^{r} \leq R_{t} .
\end{aligned}
$$

Using the well-known lagrange multiplier method, this constrained optimization problem can be converted to an unconstrained problem. The solution to (13) is identical to that of the following unconstrained problem with $R^{p}=R_{t}^{p}$ from the theory in [27],

$$
\min J_{c}=D_{c}+\lambda\left(R^{p}+R^{r}\right) .
$$

Consequently, we can achieve an optimal point on the convex rate-distortion curve for different parameters.

Once $R^{p}$ is fixed, not only the central decoded image quality but also the primary description is fixed. Hence, the change of side channel decoded image quality is dependent on the correlated information. We will explain how to generate the correlated information in the next part.

\subsection{Generating the Correlated Information $C_{i}$}

3.2.1. Correlated Information in the Approximation Subband. Because of the symmetry of both descriptions, we take description 1 as an example to explain how to get the correlated information. 


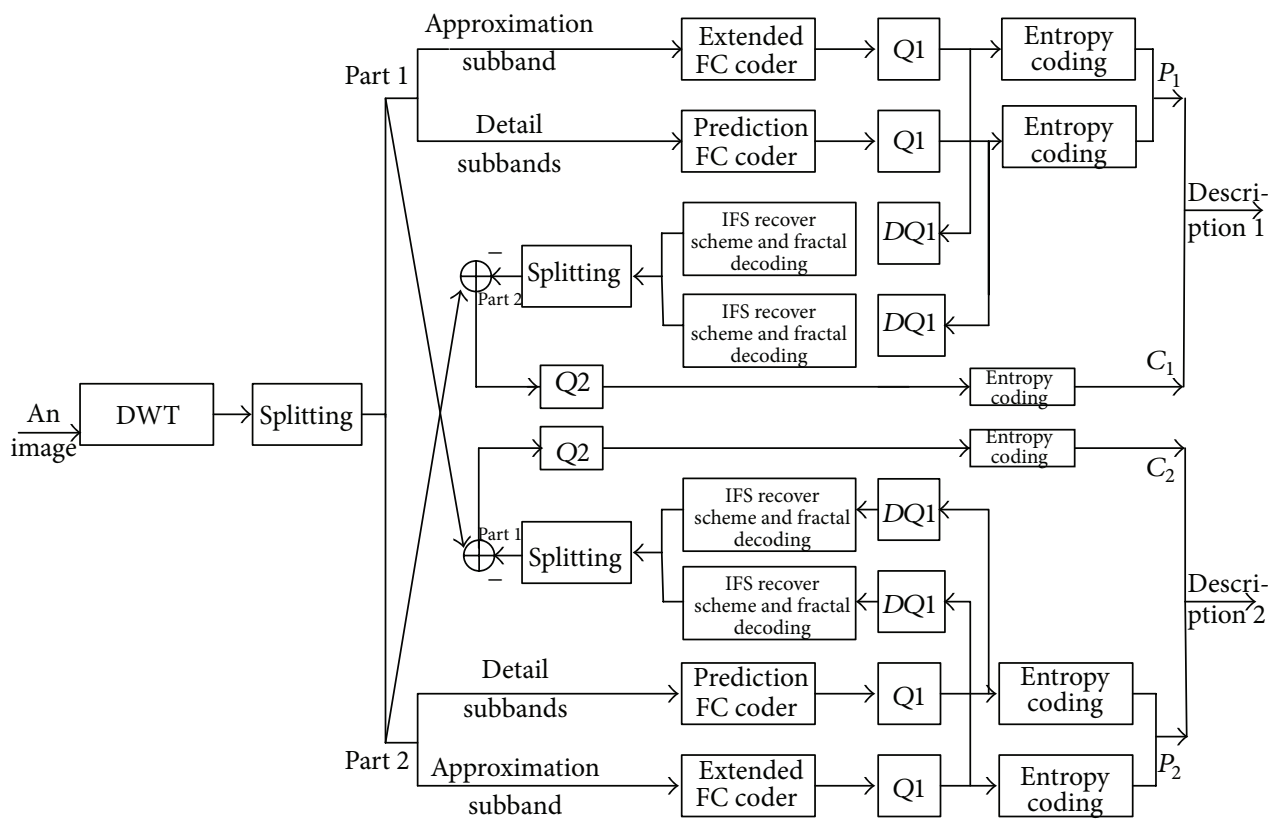

(a)

IFS recovery process

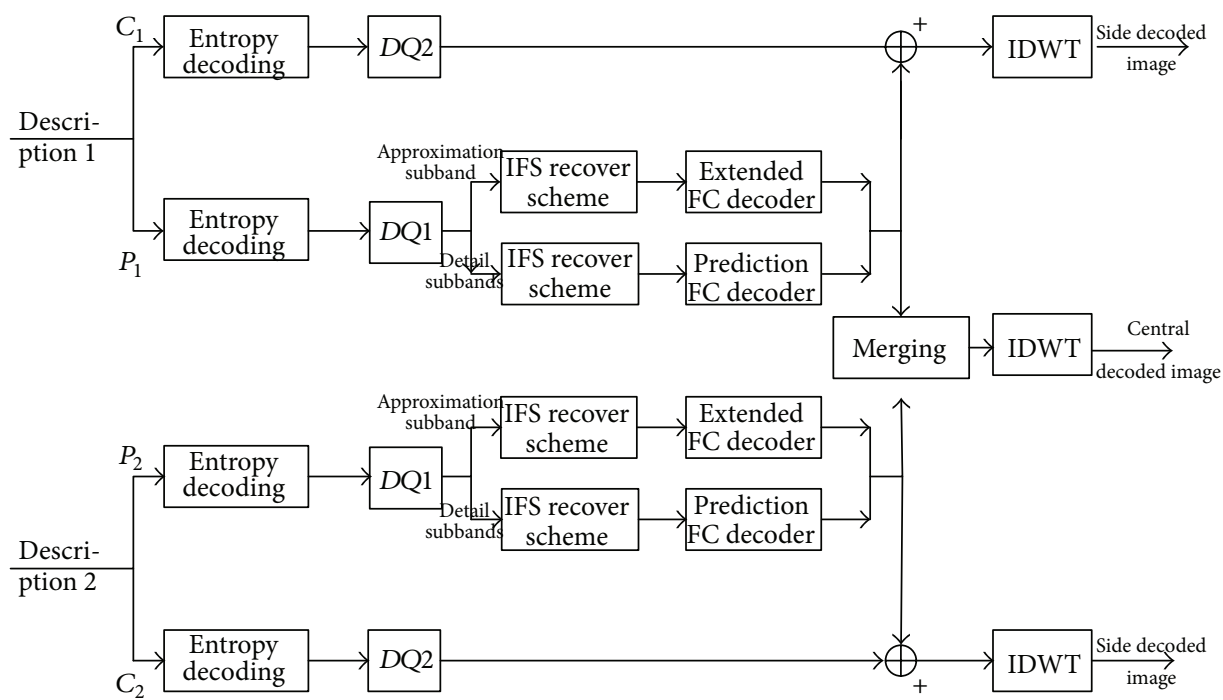

(b)

Figure 7: Process of proposed MD: (a) MD encoder, (b) MD decoder.

In Figure 2, assuming the basic block $R$ is included in description 2. When only description 1 is received, the mapping of block $R$ is lost. The mappings of basic block $R_{l}, R_{r}, R_{u}, R_{d}$ are used to estimate the lost block $R$. Therefore, some correlated information is needed at the same time. The correlated information for $R$ is quantized collage difference between $R$ and the estimation parts from the extended block of $R_{l}, R_{r}, R_{u}, R_{d}$. The difference $E_{R}$ can be expressed

$$
\begin{aligned}
E_{R}= & \left(R_{1}-\left(\omega_{u}\left(D_{u}\right)\right)_{1}\right) \cup\left(R_{2}-\left(\omega_{l}\left(D_{l}\right)\right)_{2}\right) \\
& \cup\left(R_{3}-\left(\omega_{d}\left(D_{d}\right)\right)_{3}\right) \cup\left(R_{4}-\left(\omega_{r}\left(D_{r}\right)\right)_{4}\right),
\end{aligned}
$$

where $R_{1}, R_{2}, R_{3}, R_{4}$ are four parts of $R$ and $D_{u}, D_{l}, D_{r}, D_{d}$ are the domain blocks for $R_{u}, R_{l}, R_{r}, R_{d}$ and $\omega_{u}, \omega_{l}, \omega_{d}, \omega_{r}$ are corresponding mappings from domain blocks to range blocks. $\omega(D)_{i}, i=1,2,3,4$ is the extended parts of $\omega(D)$ which are marked with red number in Figure 2. Note that the difference $E_{R}$ of block $R$ is quantized with the following:

$$
Q\left(E_{R}\right)=\operatorname{round}\left(\frac{E_{R}}{q_{\mathrm{step}}}\right),
$$

where $q_{\text {step }}$ is the quantization step, and the function round $(x)$ is used to round the $x$. 


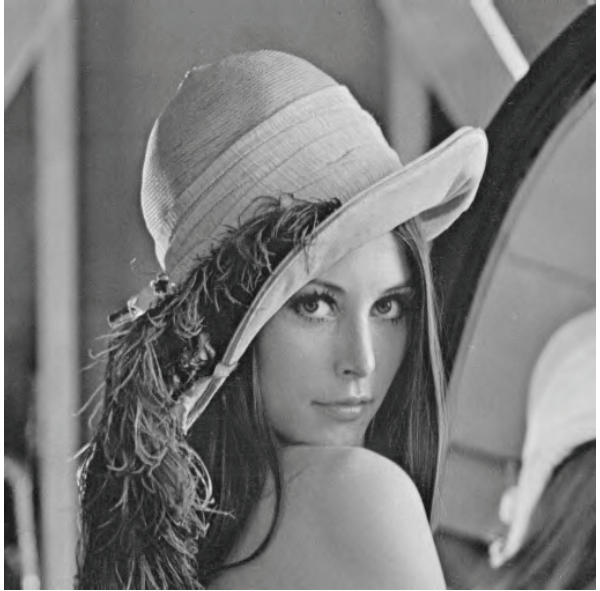

(a)

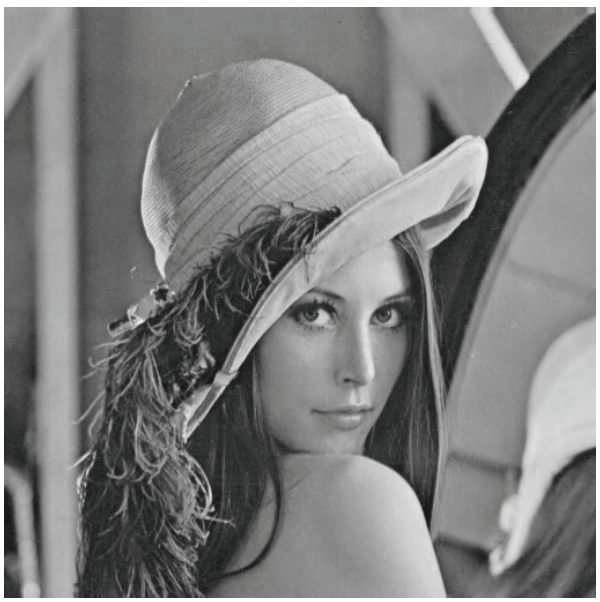

(c)

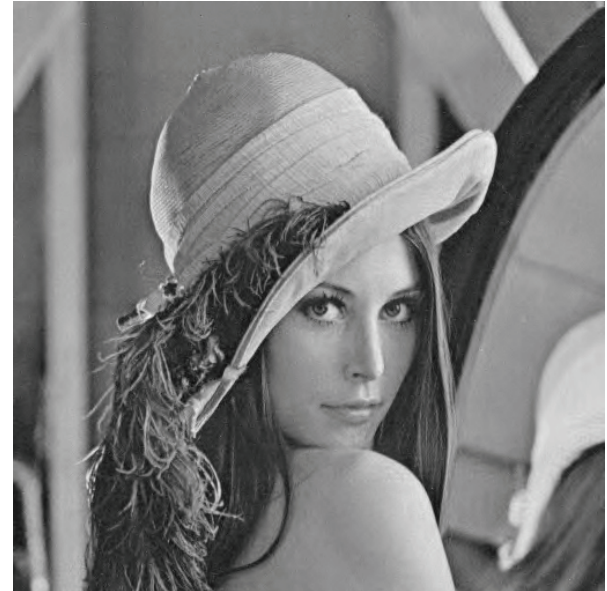

(b)

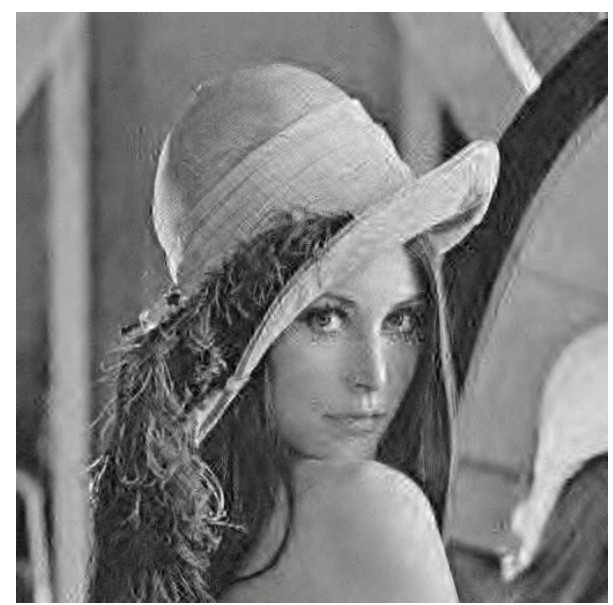

(d)

Figure 8: Sample image reconstructions, for Lena image, at $R_{t}=1.0 \mathrm{bpp}$. (a) Two channel decoder, high redundancy (PSNR: $38.22 \mathrm{~dB}$ ), (b) single channel decoder, high redundancy (PSNR: $35.00 \mathrm{~dB}$ ), (c) two channel decoder, low redundancy (PSNR: $39.40 \mathrm{~dB}$ ), and (d) single channel decoder, low redundancy (PSNR: $29.70 \mathrm{~dB}$ ).

For each basic range block in description 2, there exists the quantized difference block. All the quantized difference blocks are coded by entropy coder. The coded data is the correlated information for description 1.

3.2.2. Correlated Information in the Detail Subbands. In detail subbands, we still take description 1 as an example. In Figure 6, assuming the block range block $R$ is included in description 2 . When only description 1 is received, we need to estimate this block with the mapping of lower subband. So, there is difference between the original coefficients and the estimated coefficients just like (17). The quantized difference is the correlated information for block $R$ in description 2 . For all the blocks in description 2, we calculate this kind of difference, and all the quantized differences make up of the correlated information for description 1:

$$
E_{R}=R-\bar{\omega}\left(D_{i}^{\prime}\right), \quad Q\left(E_{R}\right)=\operatorname{round}\left(\frac{E_{R}}{q_{\mathrm{step}}}\right) .
$$

3.3. Decoding. When both descriptions are received, the primary coded information from each description is used for the reconstruction. When only one description 1 is available, the correlated information and the primary information from the description are used by the IFS recovering scheme to reconstruct the side decoded image.

\section{Experimental Results}

Two standard images $(512 \times 512)$ (Lena, Barbara) are used as the testing images, and the 10-18 Daubechies wavelet is employed for four-level subband decomposition for a $512 \times$ 512 partitioned part. For FC, the bit costs of $s_{j}$ and $o_{j}$ are chosen from 5 to 8 bits for different bit rates tested, and the size of range blocks is selected to be $8 \times 8$ or $16 \times 16$. The position of domain blocks is dependent on the domain pools. By adjusting $q_{\text {step }}$ from 8 to 65 , the bit rate of correlated information varies. 


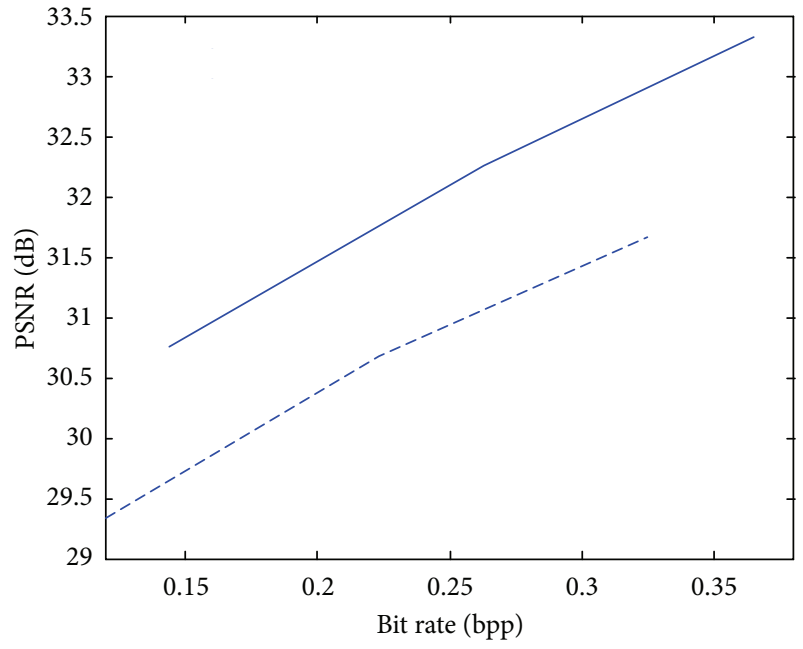

(a)

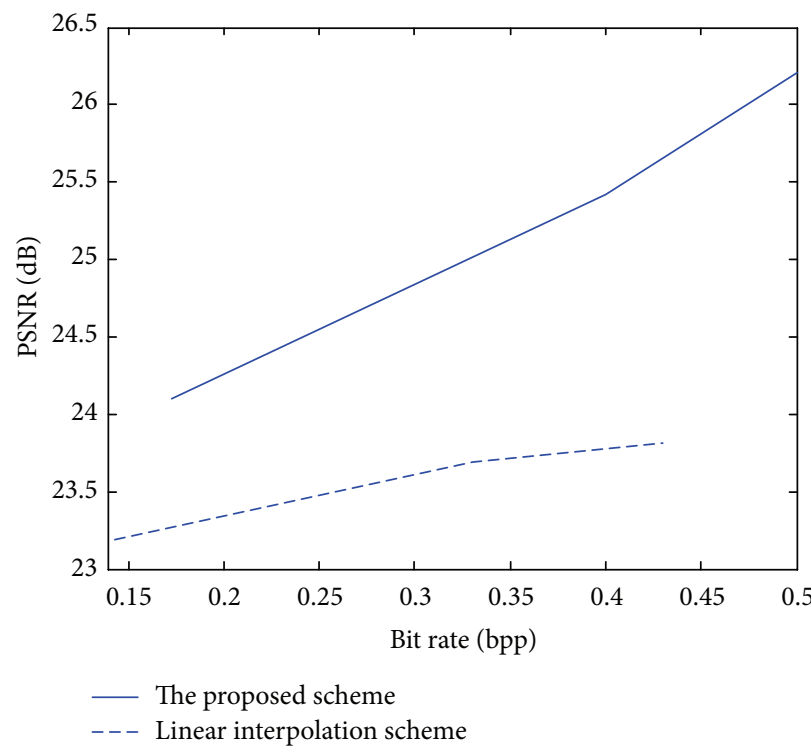

(c)

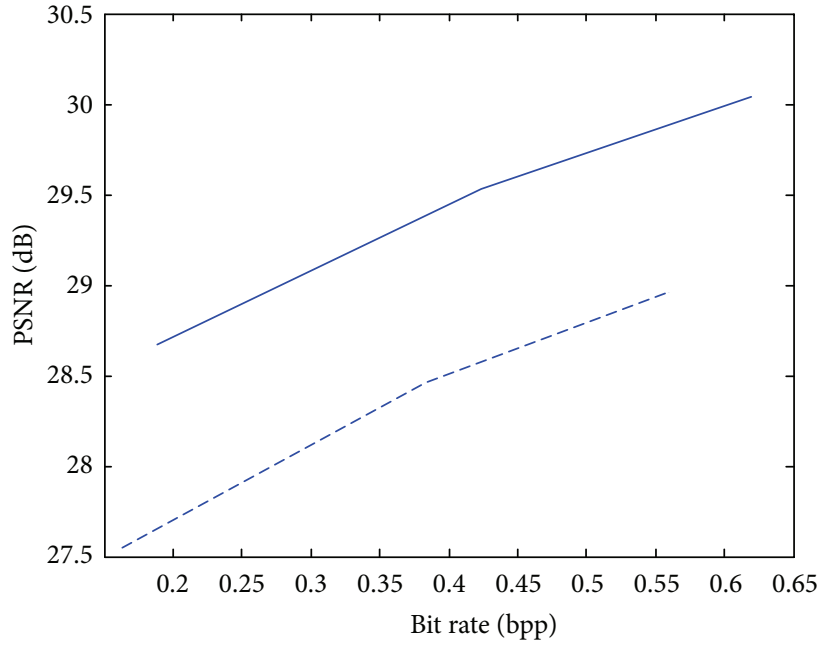

(b)

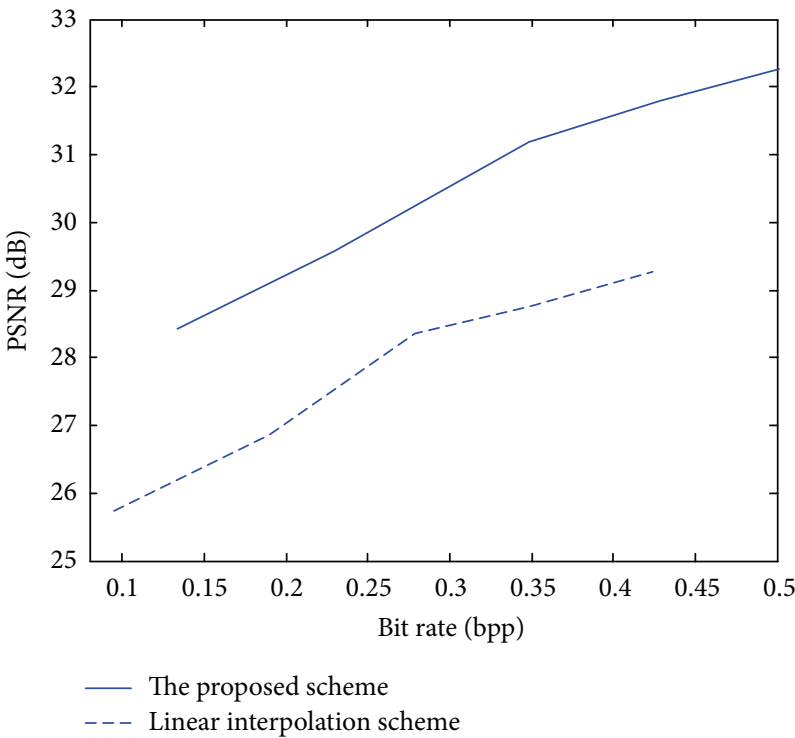

(d)

FIGURE 9: PSNR (dB) of reconstruction images using the proposed method and the Salama's linear interpolation method: (a) Zelda, (b) Elain, (c) Barbara, and (d) Lena.

4.1. MDC Performance. In the first experiment, we show performance results for our proposed MD method. The sample image reconstructions are shown in Figure 8. The test image is Lena $(512 \times 512 \times 8)$. The shown images are the reconstructed images with the target bit rate $R_{t}=1.0 \mathrm{bpp}$ in central decoder and side decoder. From Figure 9, we can see that the proposed method preserves the edge detail very well even when the redundancy of description channel is low.

Then, we evaluate the reconstruction performance of the proposed IFS recovery method. Figure 9 shows the peak signal-to-noise ratios (PSNR) of the reconstructed images for the test images "Lena," "Barbara," "Elain," "Zelda" $(512 \times 512 \times$ 8 ) by the linear interpolation method and the proposed IFS recovering scheme under different bit rates.
4.1.1. Linear Interpolation Scheme. In the linear interpolation scheme, each description only contains the primary information, corresponding to the same coding rate per description as in the proposed scheme. In this way, the redundancy between the two descriptions is minimized, which favors the central decoding performance. When only one description is received, an interpolation method used in [28] is exploited to recover the missing part.

When only one description is available, the linear interpolation method to recover the lost description yields poor performance. On the other hand, the IFS recovering scheme gives much better results. Note that the redundancy is not used in this experiment for the fair comparison. That is, in this experiment, the IFS recovering scheme and the 


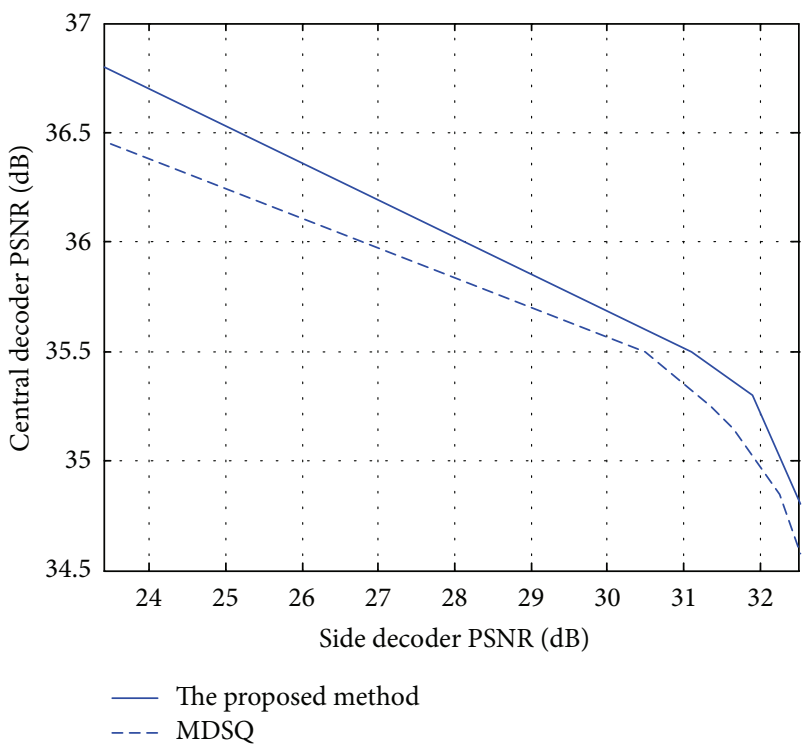

(a)

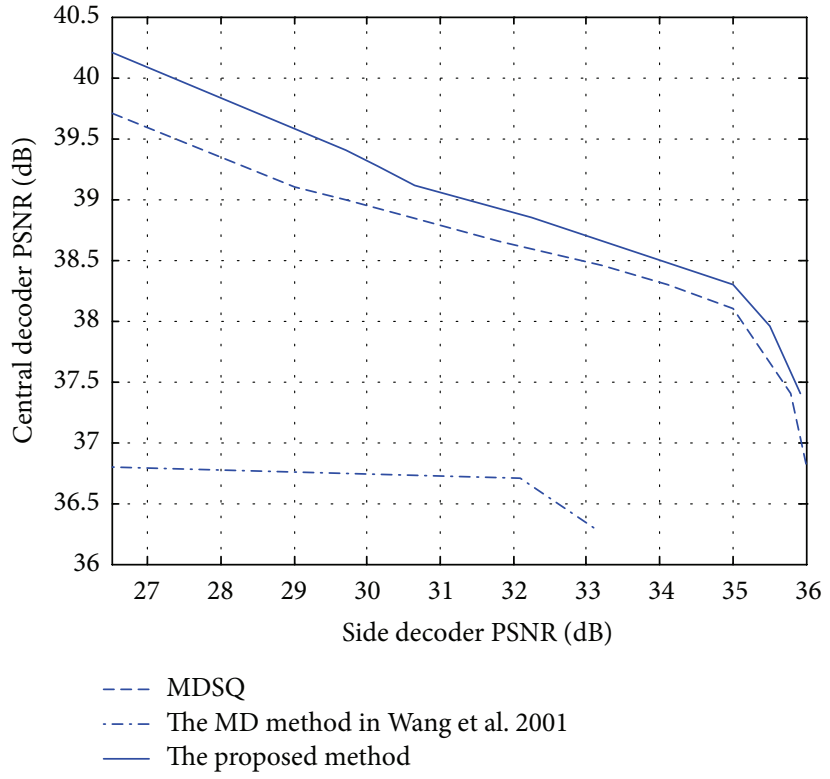

(b)

FIGURE 10: Performance comparison of Lena among MDSQ, MD method in [4] and the proposed method with target bit rates: (a) $R_{t}=0.5$ bpp, (b) $R_{t}=1.0 \mathrm{bpp}$.

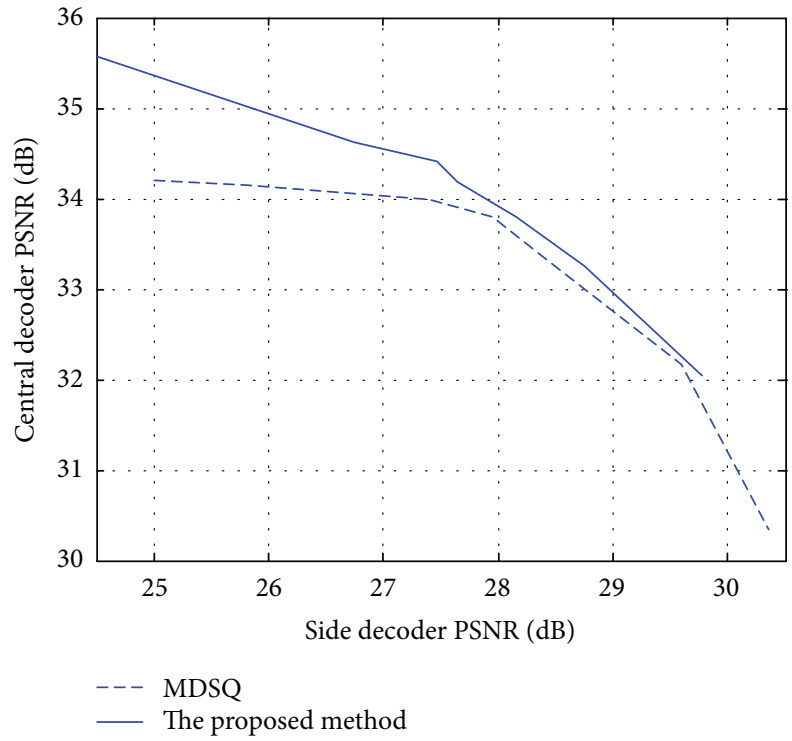

FIGURE 11: Performance comparison of Barbara between MDSQ and the proposed method with target bit rates $R_{t}=1.0 \mathrm{bpp}$.

linear interpolation scheme construct the description by only primary information. It can be seen that the proposed method is more efficient to recover the lost description than directly recovering the description using linear interpolation method.

4.2. Comparison against Other MD Image Coders. Figure 10 compares the performance attained by the proposed scheme

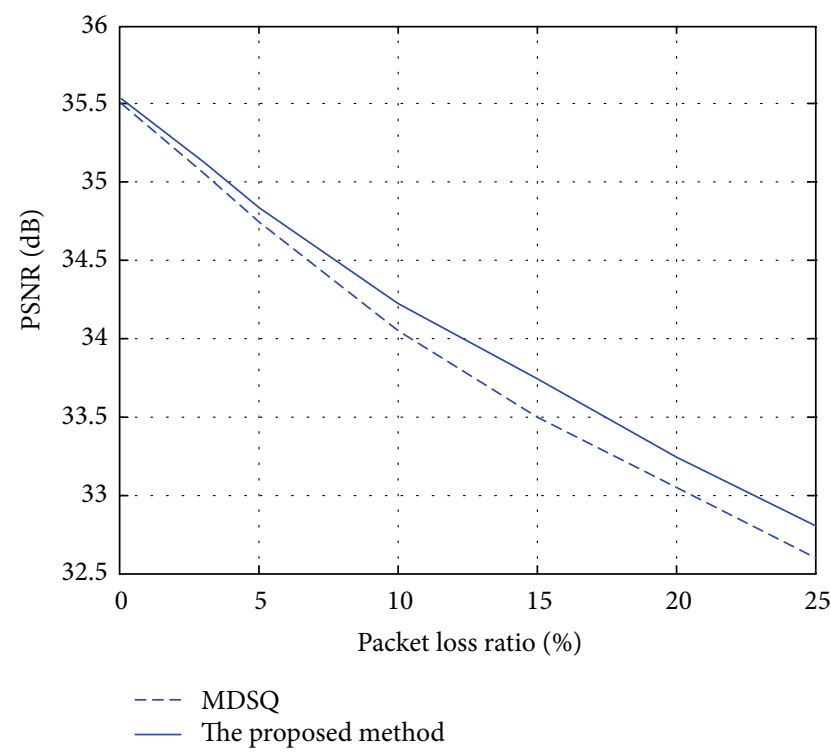

FIGURE 12: PSNR with variant packet loss ratios between MDSQ and the proposed method. $R_{t}=0.5 \mathrm{bpp}$ for the proposed method. We choose MDSQ coder with the parameters that can achieve the same central PSNR at the same $R_{t}=0.5 \mathrm{bpp}$, the test image is Lena $512 \times$ $512 \times 8$.

against MD image coders in [3, 4]. Two target bitrates, $R_{t}=0.5$ and $1.0 \mathrm{bpp}$, are tested. The $\mathrm{RD}$ curves are plotted with central reconstruction PSNR versus the average side reconstruction PSNR. This experiment is to verify the ability to achieve reasonable tradeoff points between central and side decoders with variant redundancies. For a good MD coder, 


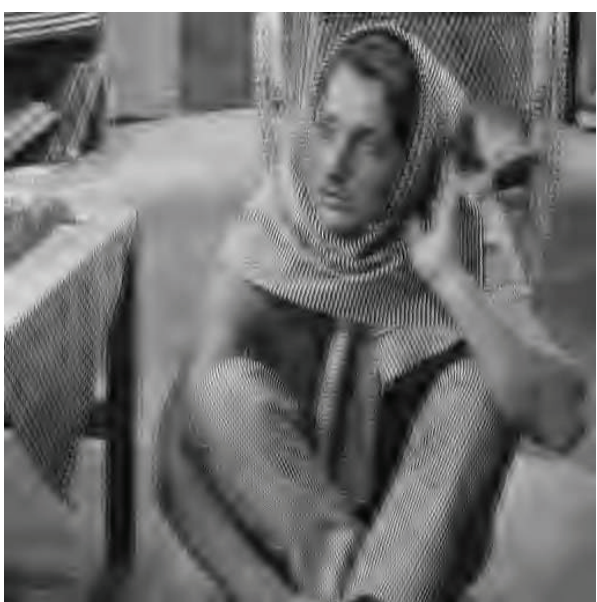

(a)

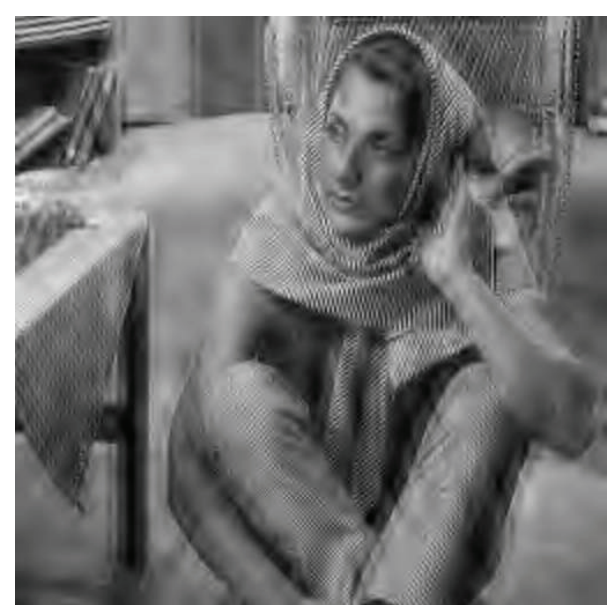

(b)

FIGURE 13: Two channel reconstruction for Barbara $(512 \times 512 \times 8)$ with packet loss ratio of $40 \%$ at $R_{t}=0.34 \mathrm{bpp}:(\mathrm{a}) \mathrm{MDSQ}$ PSNR $=22.69 \mathrm{~dB}$ and (b) the proposed method PSNR $=23.17 \mathrm{~dB}$.

it is expected to achieve higher side PSNR when the central PSNR is fixed and vice versa [6].

With the same central reconstruction distortion, the performance gap of the side decoder between the proposed scheme and MDSQ is significant, ranging from $0.4-1 \mathrm{~dB}$, which verifies the efficiency of the proposed scheme. Note that the proposed MD method has outstanding performance when the redundancy is low.

Figure 11 describes the MD coder performance for Barbara under bit rate $R_{t}=1.0 \mathrm{bpp}$. It can be seen that the proposed method can achieve better performance than MDSQ. Note that, in lower redundancy, our method can achieve larger gap than MDSQ.

In fact, the probability that a description is completely lost during the transmission is very low. Hence, it is reasonable to assume that a certain percentage of packets are lost in transmission. Each block mapping and its quanitzed difference is assumed to construct a packet. A uniformly distributed random index of the lost packet is generated for each channel. The lost packet is recovered with Salama's linear interpolation method if its estimation block used for fractal extrapolation is lost too. This experiment is tested with the packet loss ratios of $3 \%, 5 \%, 10 \%, 15 \%, 20 \%$, and $25 \%$.

The target bit rate is fixed at $0.5 \mathrm{bpp}$. The bitrate of correlated information is fixed at $0.05 \mathrm{bpp}$. The results are presented in Figure 12. The proposed method achieves same PSNR with MDSQ [3] if no packet is lost. However, PSNR of MDSQ degrades rapidly with the packet loss ratio increasing, whereas the proposed MDC proves to be more robust.

We also execute the subjective test at low bitrates. Because FC exploits the different scale redundancy of image, with $40 \%$ packet loss, the reconstructed images of the proposed MD scheme can achieve a better subjective performance of the textures and edges than MDSQ method. The results are shown in Figure 13.

\section{Conclusion}

In this paper, we have proposed an $\mathrm{MD}$ wavelet image coding scheme using fractal. This scheme exploits the ability of fractal well. Through IFS recovering scheme with the similarity in wavelet coefficient bands, the proposed scheme can reconstruct the lost information well when only one description is received. The results strongly suggest that our method can achieve better performance even when only one description is received. Furthermore, the proposed method is more robust for image transmission.

\section{Acknowledgment}

This research was supported by Science and Technology Project of Beijing Municipal Education Commission (no. KM201311232021).

\section{References}

[1] V. K. Goyal, "Multiple description coding: compression meets the network," IEEE Signal Processing Magazine, vol. 18, no. 5, pp. 74-93, 2001.

[2] V. A. Vaishampayan, "Design of multiple description scalar quantizers," IEEE Transactions on Information Theory, vol. 39, no. 3, pp. 821-834, 1993.

[3] S. D. Servetto, K. Ramchandran, V. A. Vaishampayan, and K. Nahrstedt, "Multiple description wavelet based image coding," IEEE Transactions on Image Processing, vol. 9, no. 5, pp. 813-826, 2000.

[4] Y. Wang, M. T. Orchard, V. Vaishampayan, and A. R. Reibman, "Multiple description coding using pairwise correlating transforms," IEEE Transactions on Image Processing, vol. 10, no. 3, pp. 351-366, 2001.

[5] H. Bai, C. Zhu, and Y. Zhao, "Optimized multiple description lattice vector quantization for wavelet image coding," IEEE 
Transactions on Circuits and Systems for Video Technology, vol. 17, no. 7, pp. 912-917, 2007.

[6] Y. Liu and S. Oraintara, "Feature-oriented multiple description wavelet-based image coding," IEEE Transactions on Image Processing, vol. 16, no. 1, pp. 121-131, 2007.

[7] A. Norkin, A. Gotchev, K. Egiazarian, and J. Astola, “Two-stage multiple description image coders: analysis and comparative study," Signal Processing: Image Communication, vol. 21, no. 8, pp. 609-625, 2006.

[8] T. Tillo, M. Grangetto, and G. Olmo, "Multiple description image coding based on Lagrangian rate allocation," IEEE Transactions on Image Processing, vol. 16, no. 3, pp. 673-683, 2007.

[9] Y. M. Zhou, C. Zhang, and Z. K. Zhang, "Fast hybrid fractal image compression using an image feature and neural network," Chaos, Solitons and Fractals, vol. 37, no. 2, pp. 623-631, 2008.

[10] Y.-M. Zhou, C. Zhang, and Z. K. Zhang, "An efficient fractal image coding algorithm using unified feature and DCT," Chaos, Solitons and Fractals, vol. 39, no. 4, pp. 1823-1830, 2009.

[11] J. Lu, Z. Ye, Y. Zou, and R. Ye, "An enhanced fractal image denoising algorithm," Chaos, Solitons \& Fractals, vol. 38, no. 4, pp. 1054-1064, 2008.

[12] Fractal Image Compression: Theroy and Application, Springer, New York, NY, USA, 1995.

[13] A. E. Jacquin, "Image coding based on a fractal theory of iterated contractive image transformations," IEEE Transactions of Image Processing, vol. 1, no. 1, pp. 18-30, 1992.

[14] Z. Zhang and Y. Zhao, "Improving the performance of fractal image coding," International Journal of Innovative Computing, Information and Control, vol. 2, no. 2, pp. 387-398, 2006.

[15] G. Caso and C. C. J. Kuo, "New results for fractal/wavelet image compression," in Proceedings of the SPIE, vol. 2727, pp. 536-547, 1996.

[16] R. Rinaldo and G. Calvagno, "Image coding by block prediction of multiresolution subimages," IEEE Transactions on Image Processing, vol. 4, no. 7, pp. 909-920, 1995.

[17] G. M. Davis, "A wavelet-based analysis of fractal image compression," IEEE Transactions on Image Processing, vol. 7, no. 2, pp. 141-154, 1998.

[18] T. Kim, R. E. Van Dyck, and D. J. Miller, "Hybrid fractal zerotree wavelet image coding," Signal Processing: Image Communication, vol. 17, no. 4, pp. 347-360, 2002.

[19] Y. Iano, F. S. da Silva, and A. L. M. Cruz, "A fast and efficient hybrid fractal-wavelet image coder," IEEE Transactions on Image Processing, vol. 15, no. 1, pp. 98-105, 2006.

[20] W. Xing-yuan, L. Fan-ping, and W. Shu-guo, "Fractal image compression based on spatial correlation and hybrid genetic algorithm," Journal of Visual Communication and Image Representation, vol. 20, no. 8, pp. 505-510, 2009.

[21] X. Y. Wang and S. G. Wang, "An improved no-search fractal image coding method based on a modified gray-level transform," Computers and Graphics (Pergamon), vol. 32, no. 4, pp. 445-450, 2008.

[22] Y. Zhang and X. Wang, "Fractal compression coding based on wavelet transform with diamond search," Nonlinear Analysis, vol. 13, no. 1, pp. 106-112, 2012.

[23] X. Y. Wang, Y. X. Wang, and J. J. Yun, "An improved no-search fractal image coding method based on a fitting plane," Image and Vision Computing, vol. 28, no. 8, pp. 1303-1308, 2010.

[24] W. Xing-Yuan, G. Xing, and Z. Dan-Dan, "An effective fractal image compression algorithm based on plane fitting," Chinese Physics B, vol. 21, no. 9, 2012.
[25] W. Xing-Yuan, W. Yuan-Xing, and Y. Jiao-Jiao, "An improved fast fractal image compression using spatial texture correlation," Journal of Visual Communication and Image Representation, vol. 20, no. 10, 2011.

[26] D. Semiya, T. Fujii, and M. Tanimoto, "Error resilience by using extended range blocks in fractal image coding," IEICE Technical Report 01, 2003, pp. 83-88.

[27] Y. Shoham and A. Gersho, "Efficient bit allocation for an arbitray set of quan- tizers," Acoustics, Speech and Signal Processing, vol. 2, no. 2, pp. 1445-1453, 1988.

[28] P. Salama, N. B. Shroff, E. J. Coyle, and E. J. Delp, "Error concealment techniques for encoded video streams," in Proceedings of the IEEE International Conference on Image Processing (ICIP '95), vol. 1, pp. 9-12, October 1995. 


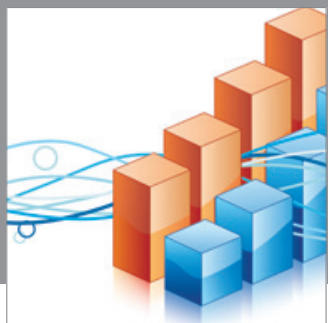

Advances in

Operations Research

mansans

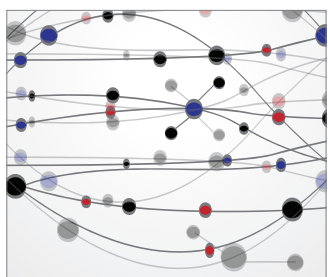

The Scientific World Journal
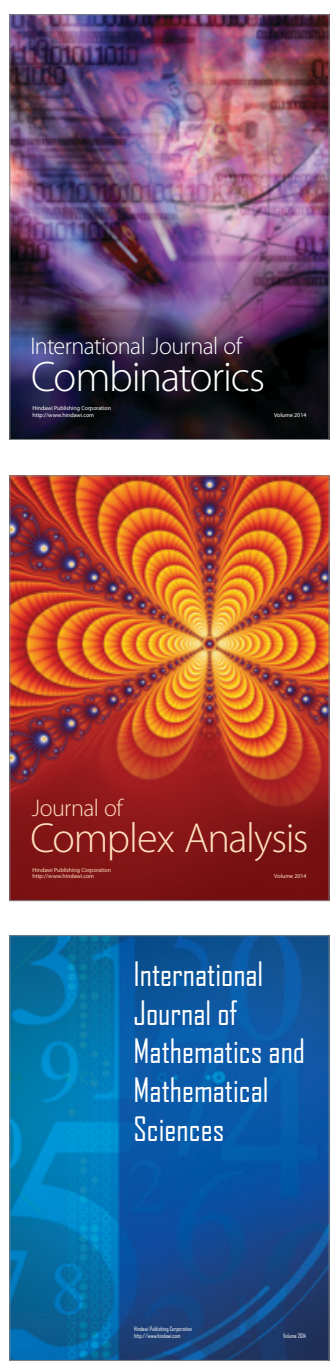
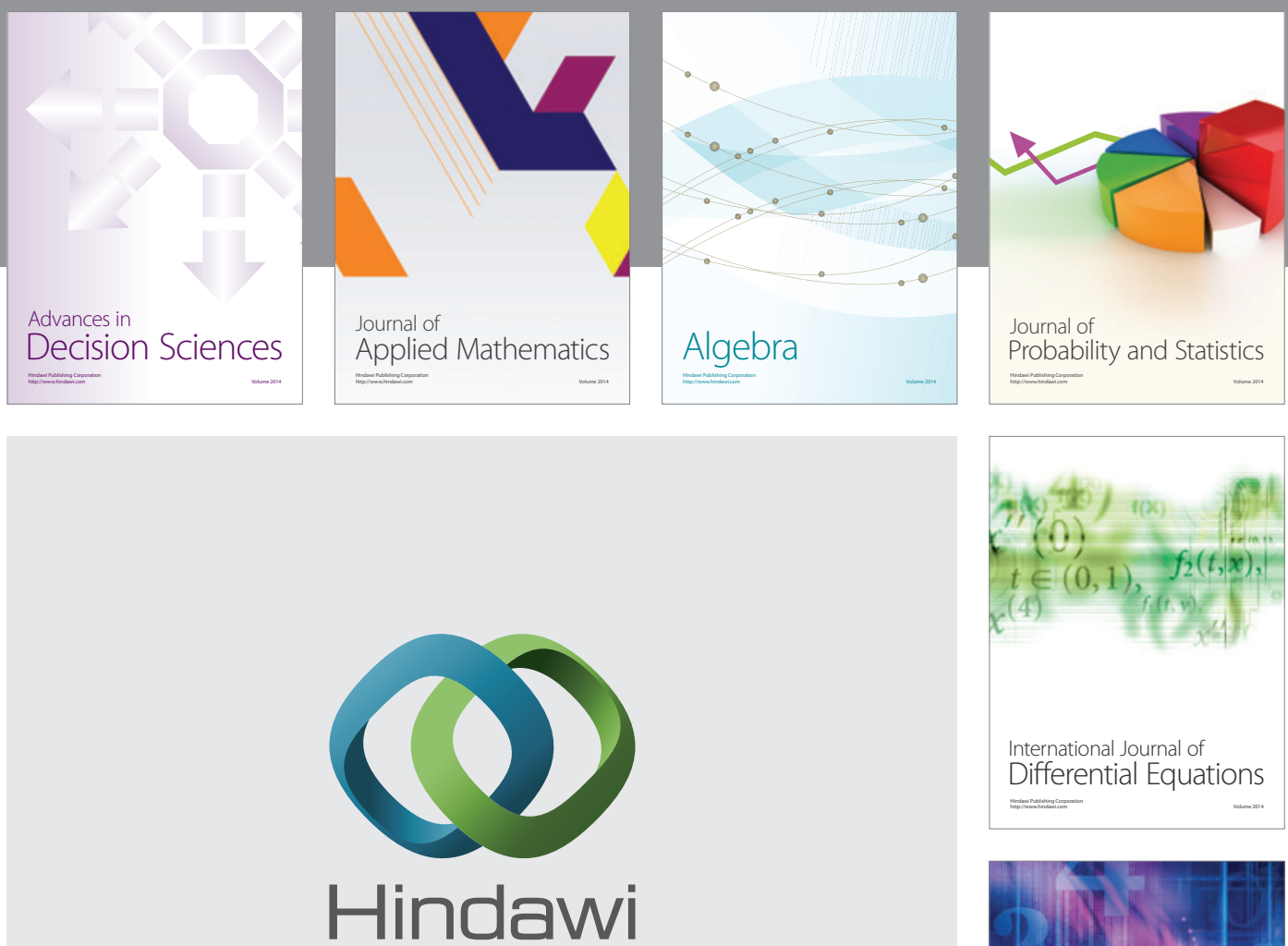

Submit your manuscripts at http://www.hindawi.com
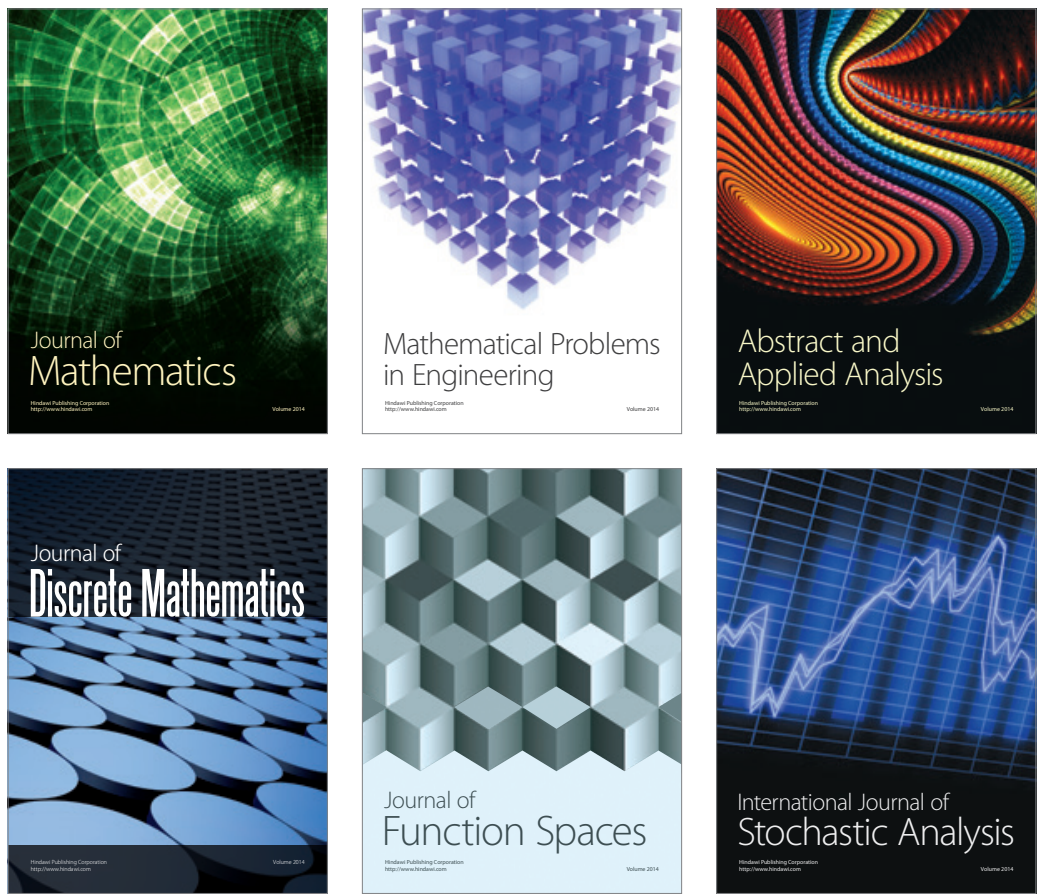

Journal of

Function Spaces

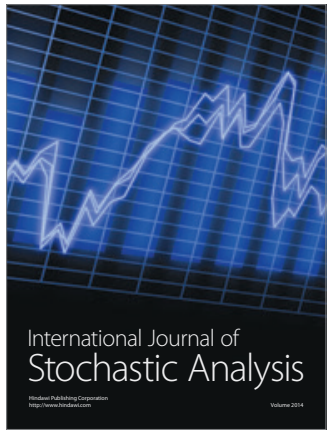

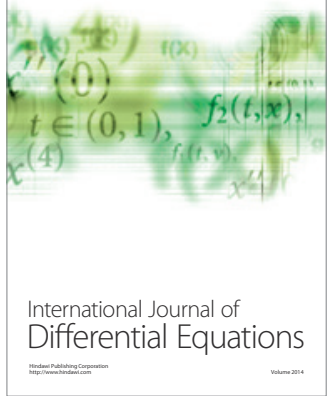
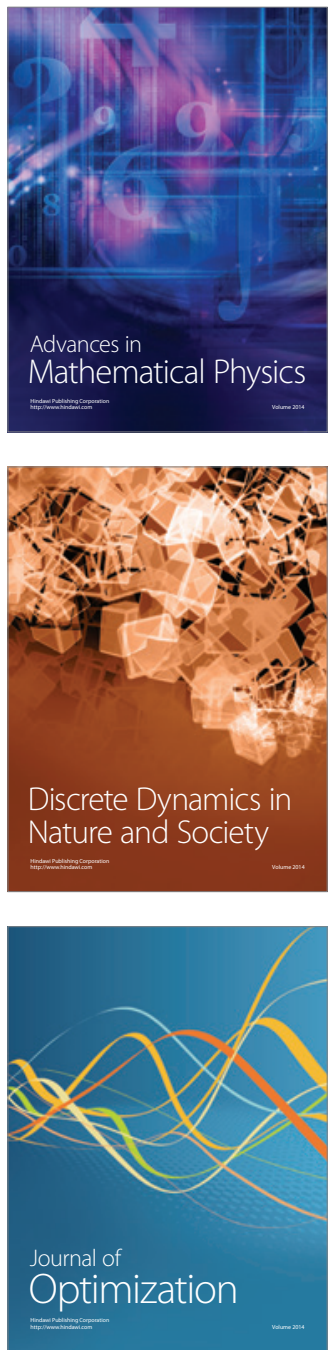